\title{
Therapeutic Administration of Mycobacterium bovis BCG Killed by Extended Freeze-Drying Modulates Airway Inflammation in a Chronic Murine Model of Asthma
}

\author{
Micheline Lagranderie, ${ }^{1,2}$, Jeroen Alfons Juliette Vanoirbeek ${ }^{3}$, Bernardo Boris Vargaftig ${ }^{4}$, \\ Pierre-Marie Guyonvarc' ${ }^{2}$, Gilles Marchal ${ }^{1,2}$, Xavier Roux ${ }^{2 *}$ \\ ${ }^{1}$ Laboratoire Immunotherapie, Institut Pasteur, Paris, France \\ ${ }^{2}$ Immunotherapix, BioTop Institut Pasteur, Paris, France \\ ${ }^{3}$ Research Unit of Lung Toxicology, Katholieke Universiteit Leuven, Leuven, Belgium \\ ${ }^{4}$ Unité de Pharmacologie Cellulaire, Institut Pasteur, Paris, France \\ Email: *roux@tolerys.com
}

Received February 1, 2013; revised March 2, 2013; accepted March 10, 2013

Copyright (C) 2013 Micheline Lagranderie et al. This is an open access article distributed under the Creative Commons Attribution License, which permits unrestricted use, distribution, and reproduction in any medium, provided the original work is properly cited.

\begin{abstract}
Background: We previously showed that treatment with Mycobacterium bovis BCG killed by extended freeze-drying (EFD BCG) modulates inflammation through regulatory $\mathrm{T}$ cells (Tregs) in an acute asthma model. In this study, we investigated the kinetics of Treg induction as well as their long-term homing in spleen and lungs correlating with reduced airway hyperresponsiveness (AHR) in a murine model of acute allergic asthma. We then evaluated the therapeutic implication of EFD BCG in a chronic asthma model. Methods: Tregs expressing Foxp3 were analyzed in various organs shortly and long-term after EFD BCG, live- and Heat Killed- (HK-) BCG treatments in an acute model of asthma. We further studied EFD BCG treatment on airway inflammation using a chronic model of asthma in mice. Results: Foxp3 expression peaked in the inguinal draining lymph-nodes (iDLNs) 2 - 4 days after EFD BCG treatment whereas it was long-term observed in spleen (days 7 to 90). This increase in Foxp3 expression was also found in lungs upon intranasal ovalbumin (OVA) challenge in OVA-sensitized mice. The loss of protection 4 months after EFD BCG treatment was correlated with the end of this phenomenon. Moreover, major lung inflammation hallmarks of severe asthma after multiple allergen challenges promoting chronic airway inflammation in OVA sensitized mice were reduced by EFD BCG treatment: AHR, eosinophils and neutrophils in bronchoalveolar lavage (BAL), mucus metaplasia, Th2 as well as Th17 cytokine levels in BAL and sera. EFD BCG treatment also enhances PPAR- $\gamma$ expression and regulates NF- $\kappa$ Bp 65 translocation in lung extracts in this model of chronic asthma. Conclusions: EFD BCG treatment induced long-term protective effect associated to Foxp3 Tregs in the spleen and lungs in an acute model of asthma and inhibits AHR in a chronic model of asthma. EFD BCG could be a new and promising immuno-modulatory alternative treatment to corticoids in severe human asthma.
\end{abstract}

Keywords: Asthma; Foxp3; Killed BCG; Modulation; Regulatory T Cells

\section{Introduction}

The hallmark of asthma is chronic moderate inflammation of the airway mucosa with transient episodes of severe inflammation and AHR [1]. In allergic asthmatic children and adults the frequency of Th2-producing cells to aeroallergens increases, emphasising their role in lung inflammation $[2,3]$. However, in the development of effective drugs to control severe allergic asthma, consideration should be given not only to disease-associated

\footnotetext{
"Corresponding author.
}

cytokines but also to eosinophilia, neutrophils and transcription factors associated to Th2 development (GATA3 ) and inflammation (NF- $\kappa$ B) $[4,5]$. Even if inhaled glucocorticosteroids are effective anti-inflammatory treatment in asthma, some patients show a poor or absent response even to high doses [6]. In smokers with asthma, agonists of PPAR- $\gamma$ produced improvements in lung function and Tregs were shown to control AHR and associated allergic disease [7,8]. New therapies enhancing PPAR- $\gamma$ and/or Tregs producing IL-10 could then represent an alternative benefit in the treatment of severe al- 
lergic asthma.

We have recently shown that Mycobacterium bovis BCG, killed by Extended Freeze drying (EFD BCG) reduced, in a mouse model, the major features of acute asthma by inducing Tregs and enhancing PPAR- $\gamma$ expression in lung cell extracts [9]. Further studies were required to investigate the kinetics of Tregs induction and their homing in various organs as well as the effect of EFD BCG treatment in a chronic model of asthma. By contrast to live and HK BCG treatments, EFD BCG long-term induced Tregs expressing Foxp3 associated with a protective effect against asthma. We showed that EFD BCG treatment administered to OVA-sensitized and multiple OVA-challenged mice significantly reduced the major hallmarks of severe asthma, enhanced PPAR- $\gamma$ expression and repressed NF- $\kappa \mathrm{Bp} 65$ in spleen and lung cell extracts.

\section{Methods}

\subsection{Treatments of Chronic and Acute Models of Asthma}

The live BCG Pasteur strain 1173P2 was grown on Sauton medium according to the conditions used for vaccine production [10]. The various BCG preparations (live $\mathrm{BCG}$, HK BCG, and EFD BCG) were obtained as previously described [11].

Male BALB/c mice (6-wk-old) were purchased from the Centre d'Élevage Janvier (Le Genest-St Isle, France) and maintained in accordance with national guidelines for animal welfare.

Acute model of asthma was previously described by Lagranderie et al. [11]. Briefly, BALB/c mice were OVA-sensitized (Valeant Pharmaceuticals, Costa Mesa, CA) and subcutaneously-treated (base of the tail) with PBS, EFD BCG, live or HK BCG after 1 OVA challenge. Twenty eight days after treatment, mice were OVAchallenged 3 consecutive days iDLNs, spleens and lungs were harvested at various times after the different treatments and stored at $-20^{\circ} \mathrm{C}$ for transcription factor measurements. AHR was measured by whole body plethysmography (EMKA technologies, Paris, France) (Enhanced pause-Penh) as previously described [11-13].

In the chronic model of asthma, mice were OVA-sensitized, EFD BCG or PBS subcutaneously-treated (base of the tail) after 1, 2 or 3 OVA challenges as described in Supplementary Figures 1(a)-(c). After the final OVAchallenges ( 3 consecutive days), AHR was measured by whole body plethysmography (Penh) and by invasive flexiVent (Scireq, Montreal, Canada), resulting in airway resistance-compliance data to methacholine (Mch) as previously described $[11,13]$. Control mice were PBStreated after the third OVA-challenge and received 3 consecutive PBS challenges before AHR determination

\section{(Supplementary Figure 1(d)).}

\subsection{Transcription Factors in Mouse Organs}

The proteins were extracted from the organs harvested at various times after treatment and stored at $-20^{\circ} \mathrm{C}$ and were resolved by $7.5 \%$ SDS-PAGE. Protein $(40 \mu \mathrm{g}$ per iDLNs and spleen, $60 \mu \mathrm{g}$ per lungs) transferred to nitrocellulose sheets were probed with mouse monoclonal anti-Foxp3, T-bet and GATA-3 (Santa Cruz Biotechnology, Santa Cruz, CA) or $\beta$-actin mouse mAb (Ac-15 Abcam, Cambridge, UK). HRP-conjugated polyclonal goat anti-rabbit (DakoCytomation, Glostrup, Denmark) or goat anti-rabbit IgG (Santa Cruz Biotechnology) were used as secondary Abs. The immune complex was visualized and scan-analyzed as previously described [9].

In the chronic model of asthma, spleen and lung nuclear extracts $(10 \mu \mathrm{g})$ were tested for NF- $\kappa$ B activation or PPAR- $\gamma$ expression with NF- $\kappa$ Bp65 or PPAR- $\gamma$ TransAM transcription factor assay kits (Active Motif, Carlsbad, CA) according to the manufacturer's recommendation.

\subsection{Bronchoalveolar Lavage (BAL) Fluid, Differential Cell Counts and Cytokine Measurement}

Twenty-four hours after the final challenges, mice were anesthetized, the trachea was cannulated and lungs were washed with PBS $(500 \mu 1 \times 3)$. After centrifugation the supernatants were stored at $-20^{\circ} \mathrm{C}$ for cytokine analysis and the individual pellets were suspended in $500 \mu \mathrm{l}$ of PBS and the total bronchoalveolar lavage cells were counted. Cytospins were prepared and stained with DiffQuick (Baxter Dade AG, Duedingen, Switzerland) for differential cell counts.

Cytokine contents in the BAL and sera were determined using Bio-Plex Cytokine Assay (Bio-Rad, Marnes La Coquette, France) [11]. The sensitivities of the cytokines tested were, $0.2 \mathrm{pg} / \mathrm{ml}$ (IL-6), $0.3 \mathrm{pg} / \mathrm{ml}$ (IL-5, KC), $0.4 \mathrm{pg} / \mathrm{ml}$ (IL-10), $0.8 \mathrm{pg} / \mathrm{ml}$ (IL-17), $1.2 \mathrm{pg} / \mathrm{ml}$ (IFN- $\gamma$ ), $1.4 \mathrm{pg} / \mathrm{ml}$ (TNF- $\alpha$ ), $12 \mathrm{pg} / \mathrm{ml}$ (IL-13), $15 \mathrm{pg} / \mathrm{ml}$ (eotaxin).

\subsection{Lung Histology}

In the chronic model of asthma, the lungs from different groups of mice were recovered 24 hours after the final OVA-challenges and fixed in $10 \%$ buffered formalin. After paraffin-embedding, tissue sections were stained with either hematoxylin/eosin (HE) or periodic acidSchiff (PAS).

\subsection{Statistical Analysis}

The mean and standard error for each group of six mice were calculated. The Instat package from GraphPad (San Diego, CA) was used for analysis with the Student $t$ test. 


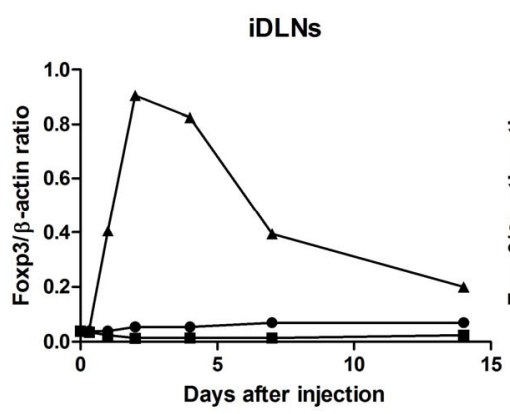

(a)

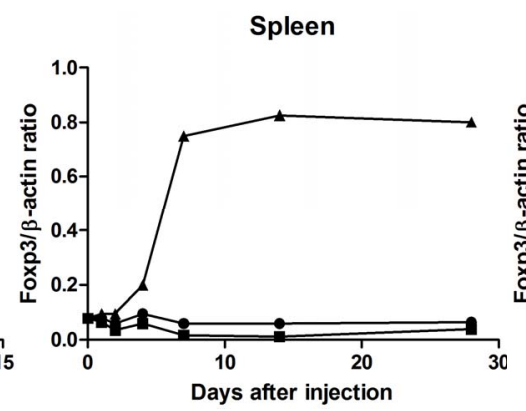

(b)

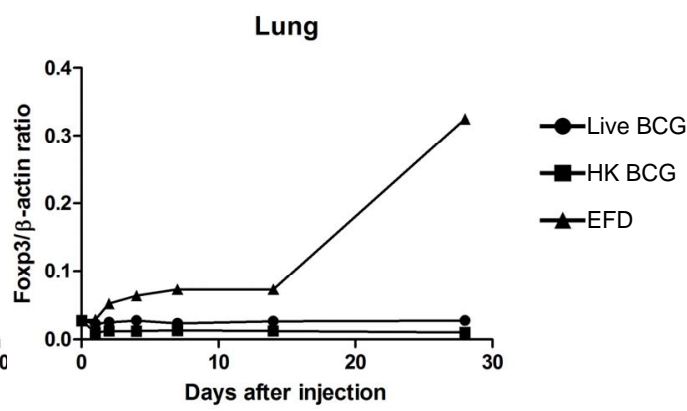

(c)

Figure 1. Only EFD BCG treatment increases Foxp3 expression. Mice were OVA sensitized and Foxp3 expression was measured in extracts from inguinal lymph nodes (a) spleens (b) and lungs (c) at various times after treatment with live, HK and EFD BCG and 24 hours after the OVA challenge (day 28). Six mice per group.

\section{Results}

\subsection{Kinetics Induction and Long-Term Homing of Tregs in Various Organs after EFD BCG Injection in OVA-Sensitized Mice}

We have previously shown that EFD BCG subcutaneously injected to mice induced the recruitment of plasmacytoid dendritic cells (pDCs) in the iDLNs [9]. These pDCs promoted, few days after the EFD BCG injection, the differentiation of naïve $\mathrm{T}$ cells towards Tregs expressing Foxp3.

However, the kinetics and the long-term homing of Tregs in various organs remained to be studied from the EFD BCG injection to OVA-sensitized mice until 24 hours after the OVA challenge. As compared to live or HK BCG injections only EFD BCG injection induced a significant increase of Foxp3 expression whatever the organ studied (Figures 1(a)-(c)). In the iDLNs, Foxp3 expression slightly increased at day 1 after EFD BCG injection, peaked at days 2 - 4 and then decreased regularly (Figure 1(a)). Concomitantly to decreased expression of Foxp3 in the iDLNs we observed an increase of Foxp3 expression in the spleen that peaked at day 14 and remained at high levels even 24 hours after the OVA challenge (day 28) (Figure 1(b)). In the lungs, the levels of Foxp3 expression slightly increased until day 14 and were strongly enhanced 24 hours after the OVA-challenge (Figure 1(c)).

It has to be noted that even though the protein content used for lung extracts $(60 \mu \mathrm{g})$ is higher than that of iDLNs and spleen extracts $(40 \mu \mathrm{g})$ we found lower levels of Foxp3 expression in the lungs probably due to a lower number of $T$ cells in lung tissue extracts. We have previously shown that the $\mathrm{pDCs}$ recruited to the iDLNs after EFD BCG injection polarize naïve $T$ cells towards Tregs expressing Foxp3 [9] and the present results suggested that these Tregs expanded and migrated to the spleen where they homed before migrating to the lungs 24 hours after the OVA-challenge.

We then studied the long-term protective effect of
EFD in an acute model of asthma in which mice were OVA-sensitized, EFD BCG-treated and, OVA-challenged 2, 3 or 4 months after the treatment. The EFD BCG-treated as compared to PBS-treated mice were protected at least 3 months as shown by reduced Penh values at 2 and 3 months $(P<0.001)$, at 4 months the Penh values of EFD BCG-treated mice were closed to those of PBS-treated mice $(\mathrm{P}<0.05)$ (Figures 2(a)-(c)). The capacity of EFD BCG to reduce AHR is correlated to the expression of Foxp3 in spleens and lungs (Figures 2(d)(f)). When we observed a decreased Foxp3 expression in both compartments (4 months after the EFD BCG treatment) the AHR was not modulated (Figures 2(a)-(f)).

\subsection{Treatment with EFD BCG Reduces AHR and Bronchial Inflammation in a Model of Chronic Asthma}

BALB/c mice were OVA-sensitized and OVA-challenged as shown in Supplementary Figures 1(a)-(d). The effect of EFD BCG treatment administered one week after 1, 2 or 3 OVA-challenges was determined by measuring AHR 24 hours after the final OVA-challenges (3 consecutive days). In PBS-treated mice, after 1 or 2 and particularly after 3 OVA-challenges, the Penh values in response to various doses of $\mathrm{Mch}$, increased significantly ( $\mathrm{P}<0.001$ ), as compared to control mice receiving PBS as final challenges, (Figure 3(a)). By contrast, EFD BCG-treated mice, even those treated after 3 OVA challenges had significant lower Penh values than PBStreated and OVA-challenged mice $(\mathrm{P}<0.001)$ (Figure 3(a)). Mice EFD BCG treated after 1, 2 or 3 OVA challenges displayed similar Penh values than control mice PBS-treated and PBS-challenged (Figure 3(a)). The measurement of dynamic compliance and resistance in mechanically ventilated mice induced a significant change in responsiveness to Mch in EFD BCG-treated mice after 3 OVA-challenges compared with PBS-treated mice ( $\mathrm{P}<$ 0.001 and $\mathrm{P}<0.01$ respectively) (Figures 3(b) and (c)). 


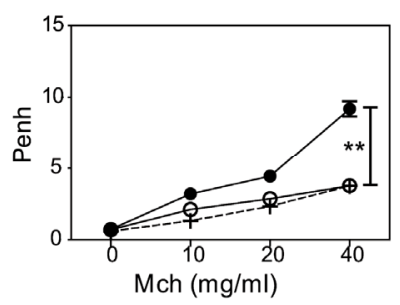

(a)

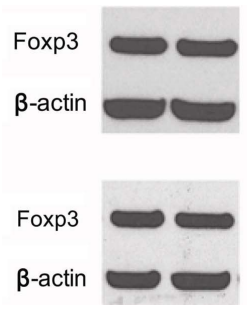

(d)

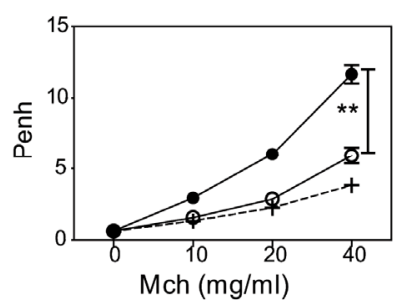

(b)

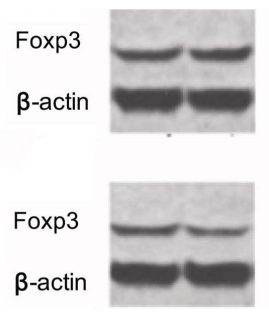

(e)

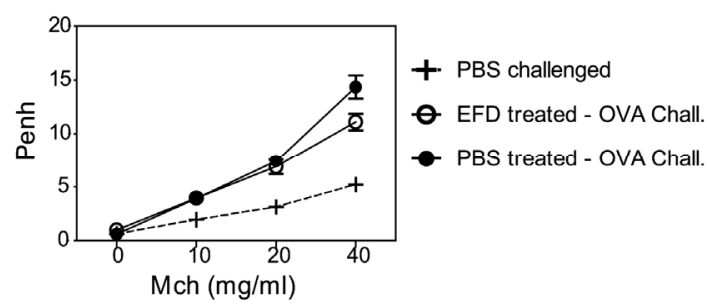

(c)

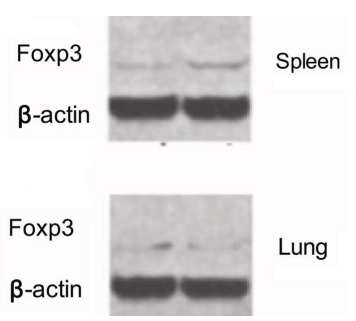

(f)

Figure 2. EFD BCG treatment induced long-term Foxp3 expression correlating with protection. OVA-sensitized mice were PBS- or EFD BCG-treated. Twenty-four hours after OVA- or PBS-challenges the Penh values were recorded after increasing doses of Mch (a) 2; (b) 3 and (c) 4 months after the various treatments. Six mice per group. Foxp3 expression was measured in lung cell extracts recovered (d) 2; (e) 3 and (f) 4 months after the EFD BCG treatment. Each band represents lung protein extracts of 3 mice. ${ }^{*} \mathbf{P}<0.1,{ }^{* * *} \mathrm{P}<0.001$.

The number of lymphocytes, eosinophils and neutrophils of PBS-treated mice increased in BAL after 3 OVA challenges, whereas EFD BCG-treatment (after 1, 2, or 3 OVA challenges) reduced significantly these cell numbers $(\mathrm{P}<0.001)$ (Figures 4(a)-(c)). The number of macrophages was not affected by the OVA challenges in PBS-treated mice, only mice EFD BCG treated after the first OVA challenge showed a slight decrease of macrophages in the BAL $(\mathrm{P}<0.01)$ (Figure 4(d)).

As compared to control mice (Figure 4(e)), histologic studies showed in PBS-treated mice numerous infiltrating cells in the lung parenchyma in twice-challenged mice (data not shown) and an inflammation in those third-challenged (Figure 4(f)). EFD BCG administered after the third OVA-challenge reversed this inflammatory process (Figure $4(\mathrm{~g})$ ). Similarly, the number of mucus goblet cells were significantly increased after PBS than after EFD BCG treatment $(80 \%$ vs. $25 \%$ in small bronchi and $80 \%$ vs. $45 \%$ in large bronchi after 3 OVAchallenges).

\subsection{EFD BCG Treatment Reduces Inflammatory Cytokines in Sera and BAL Fluid}

Levels of Th1, Th2 and Th17 cytokines and eotaxin, a specific eosinophil chemoattractant, were measured in sera and BAL fluids of all groups of mice. In both compartment, as compared to PBS treatment EFD BCG administered after one (Figures 5(a) and (b)), two (Figures 5(a) and (d)) or three (Figures 5(e) and (f)) OVA challenges reduced similarly Th2 (IL-5, IL-13), inflammatory (KC, IL-6), and Th17 (IL-17) cytokines.
Eotaxin levels particularly increased in BAL fluid of PBS-treated mice after the second and third challenges were strongly reduced by EFD BCG treatment. TNF- $\alpha$ levels were only detected in the sera and were significantly reduced in EFD BCG-treated mice, whereas IFN- $\gamma$ and IL-10 levels increased in sera and BAL fluids of EFD BCG-treated mice. It has to be noted that EFD BCG even when administered after 3 OVA challenges reduced inflammatory cytokines in sera and BAL fluids at similar levels than controls PBS challenged (Figures 5(e) and (f)).

\subsection{Enhanced Foxp3 Expression Is Associated to Reduced NF- $\kappa$ B Activation in Spleen and Lungs}

We have previously shown in a model of acute asthma that EFD BCG induced Tregs expressing Foxp3 [9] increased T-bet and reduced GATA-3 transcription factor signatures of Th1 and Th2 immune responses respectively [14]. Thus we measured these transcription factors in lung cell extracts of mice EFD BCG or PBS treated after 1, 2, or 3 challenges. As shown in Figure 6(a), after the last OVA-challenge, as compared to PBS-treated mice, GATA-3 expression is decreased in the lungs extracts of EFD BCG-treated mice whereas Foxp3 expression is increased. EFD BCG treated mice displayed higher levels of T-bet expression in their lung extracts than PBS-treated and OVA-challenged mice. It has to be noted that T-bet expression in the lungs of PBS-treated and PBS-challenged control mice is identical to that of EFD BCG-treated mice (Figure 6(a)).

Same profiles were observed in spleen cell extracts 


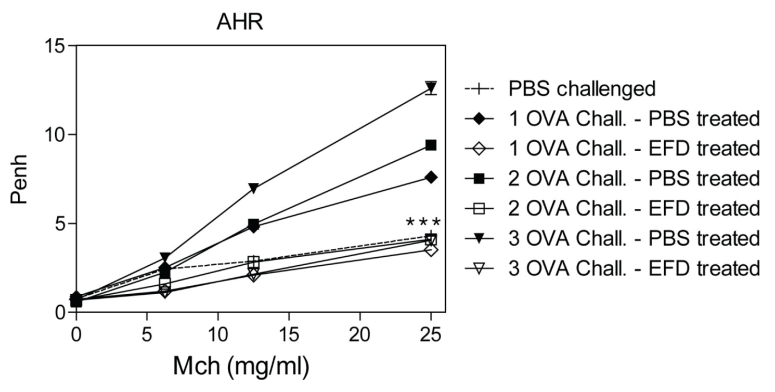

(a)

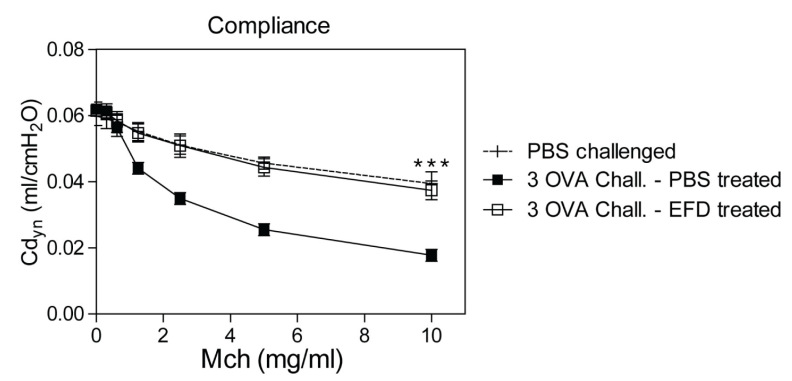

(b)

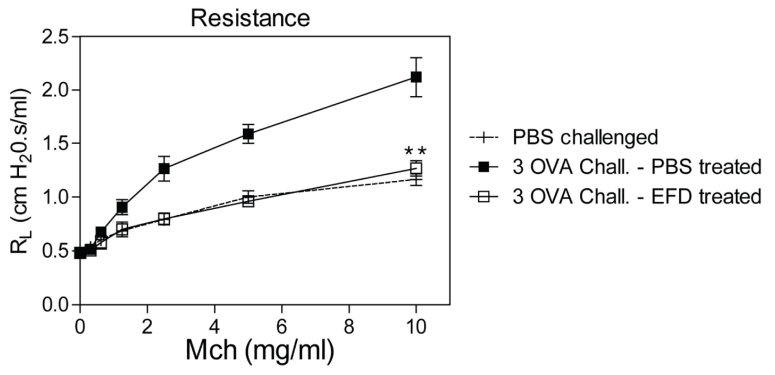

(c)

Figure 3. EFD BCG treatment reduces AHR in a chronic model of asthma. OVA-sensitized mice (6 per group) were EFD BCG treated after 1, 2 or 3 OVA challenges and OVA or PBS-challenged (supplementary Figure 1). (a) Twentyfour hours after the last OVA-challenges Penh values were recorded after increasing doses of Mch; (b), (c) Changes in lung compliance and resistance of EFD BCG- or PBStreated mice after 3 OVA challenges are shown. ${ }^{* *} \mathbf{P}<0.01$, ${ }^{* * *} \mathbf{P}<\mathbf{0 . 0 0 1}$.

(data not shown).

As previously shown in an acute model of asthma [9] we observed, as compared to PBS-treated mice, a blockade of NF- $\kappa \mathrm{Bp} 65$ translocation to the spleen and lung nucleus (Figure 6(b)) of EFD BCG-treated mice after 1, 2 , or 3 OVA-challenges. The expression of PPAR- $\gamma$ increased significantly $(\mathrm{P}<0.001)$ in spleen and lung nuclear cell extracts when mice were EFD BCG treated after 1 and 2 OVA-challenges and this increase was less marked after 3 OVA-challenges $(\mathrm{P}<0.01)$ (Figure 6(c)).

\section{Discussion}

We recently showed that EFD BCG treatment reduces inflammation in an acute model of asthma $[9,11]$ as well as in models of acute and chronic colitis [15] with no side effects observed in mice. The major finding of the present investigation is that EFD BCG treatment after multiple allergen challenges in a chronic model of asthma showed comparable reduction in lung inflammation than observed in the acute model previously described [9,11]. Indeed, administration of EFD BCG after the onset of acute eosinophilic airway inflammation was found to decrease cellular inflammation (eosinophils, lymphocytes, neutrophils) in BAL during chronic allergen challenges. Moreover, therapeutic treatment with EFD BCG after 3 OVA-challenges significantly decreased the size and extent of inflammation in lungs and reduced the number of mucus producing goblet cells within the bronchiolar epithelium. The decrease in lung inflammation observed after EFD BCG treatment correlated with lowered inflammatory cytokines (Th1, Th2 and Th17) and increased IL-10 and IFN- $\gamma$ production levels in BAL and sera. Moreover we showed that the long-term presence of Tregs expressing Foxp3 in spleen and lungs correlated with reduced AHR in an acute model of asthma.

Patients with severe refractory asthma fail to show a clinical improvement in lung function in response to high doses of inhaled steroids $[6,16]$. These individuals have a marked defect in the capacity of their CD4 $+\mathrm{T}$ cells to synthesize IL-10 in response to glucocorticoids in vitro compared with their glucocorticoid-sensitive asthmatic counterparts [17]. IL-10 is an important alternative therapeutic candidate for the treatment of asthma and clinical trials using recombinant IL-10 showed that it is well tolerated [8]. However a major disadvantage of using IL-10 is that it has a relatively short half-life in vivo $[8,18]$. Thus, therapeutic treatment with EFD BCG that induced IL-10-secreting Tregs [9] long term homing in spleen and that deliver immuno-regulatory signals following challenge with allergens seemed a more attractive strategy. Indeed, we found high levels of IL-10 in the sera and BAL fluids of EFD BCG treated mice as compared to those PBS-treated in the model of chronic allergic asthma after 1, 2 or 3 OVA-challenges.

In mice models, live and HK BCG suppressed airway eosinophilia largely through IFN- $\gamma$ production [19-21] and HK Listeria prevented allergy through induction of Tregs producing IFN- $\gamma$ and IL-10 [22]. We have previously shown [11] that lung explants of EFD BCG treated mice produced less IFN- $\gamma$ and more IL-10 than those of mice treated with live or HK BCG; in the present study we observed an increase of both cytokines after EFD BCG as compared to PBS treatment particularly after 3 OVA challenges.

The increased levels of IFN- $\gamma$ shown in this study could be due to the model of asthma studied (chronic vs. acute), the mouse strain (BALB/c vs. BP2 more Th2 


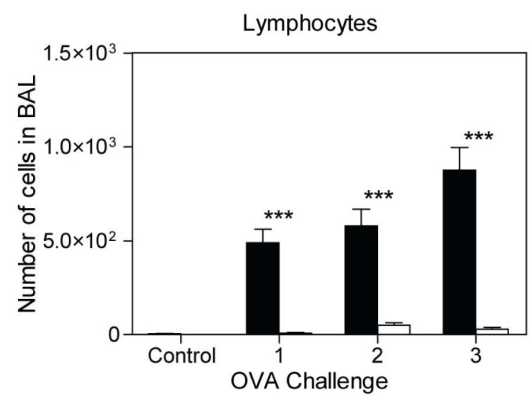

(a)

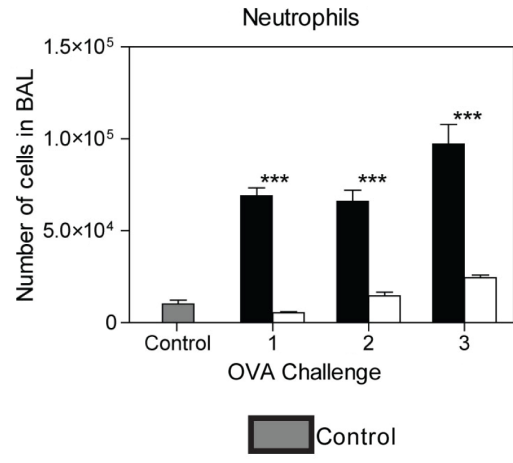

(c)

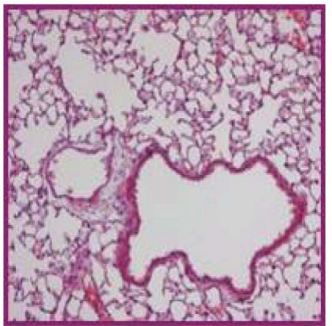

(e)

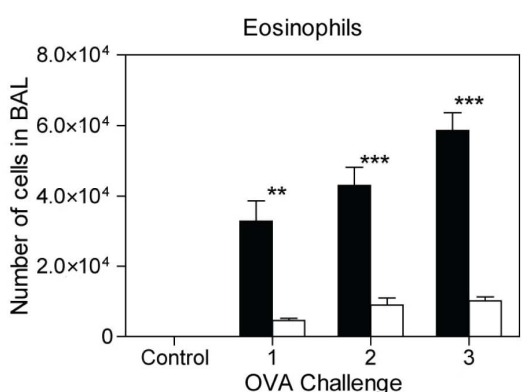

(b)

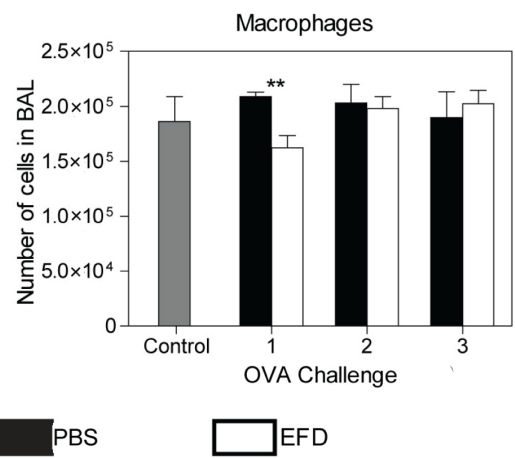

(d)

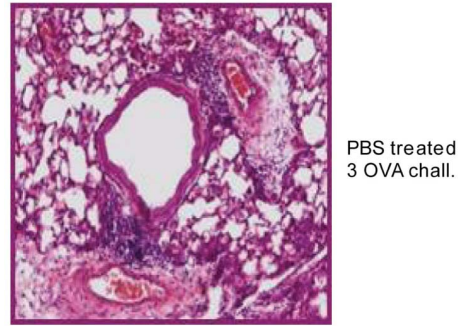

(f)

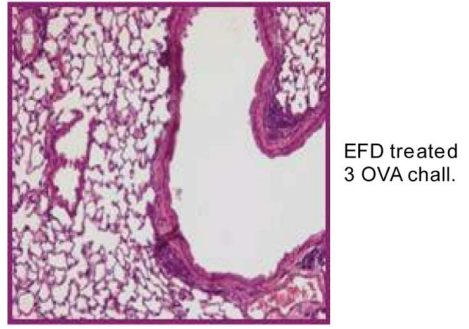

(g)

Figure 4. EFD BCG treatment reduces lung inflammation in a chronic model of asthma. (a)-(d) Inflammatory cells in BAL of EFD BCG-treated mice after 1, 2, or 3 OVA-challenges. Lung histological sections stained with hematoxylin and eosin from mice treated after 3 OVA challenges are shown (e) PBS-treated and PBS-challenged control mice, (f) PBS-treated and OVA-challenged mice, (g) EFD BCG-treated and OVA-challenged mice. ${ }^{* *} \mathrm{P}<0.01,{ }^{* * *} \mathrm{P}<0.001$.

prone) and finally the compartment chosen for the dosage (serum and BAL vs. lung explants). The various models of acute or chronic inflammation in which mice were EFD BCG treated $[9,15]$, we have always observed an increased expression of T-bet, which is the transcription factor signature of Th1 immune response, in comparison with PBS-treated mice [23]. In this study we observed sustained levels of IFN- $\gamma$ after EFD BCG treatment compared to PBS-treated mice, suggesting that despite its immunoregulatory properties EFD BCG treatment could maintain an efficient immunocompetence of the host via the Th1 arm of immunity.

Inhibitors of NF- $\kappa$ B have shown efficacy in animal models of inflammatory diseases, however these drugs have been associated with side effects and toxicity [16]. We have previously shown that EFD BCG blocks NF- $\kappa$ B translocation to the nucleus [9] without known side ef- fects even when administered at high doses (manuscript in preparation). It has been shown that activation of PPAR- $\gamma$ in dendritic cells inhibits eosinophilic airways [24] and lead to inhibition of NF- $\kappa$ B activation [25]. Thus EFD BCG a strong inhibitor of NF- $\kappa$ B and enhancer of PPAR- $\gamma$ expression, even if the treatment occurred after established inflammation, could be an effective treatment in chronic asthma.

A study showed that the number of Tregs was decreased in lungs but not in peripheral blood of asthmatic children, and they were restored following inhalation of corticosteroids [26]. However, treatment with corticosteroids has been also shown to inhibit induction of IL-10 and development of Tregs [17].

Improved care of severe refractory asthma is a major unmet medical need calling for novel therapeutic options controlling airway inflammation over a long period of 


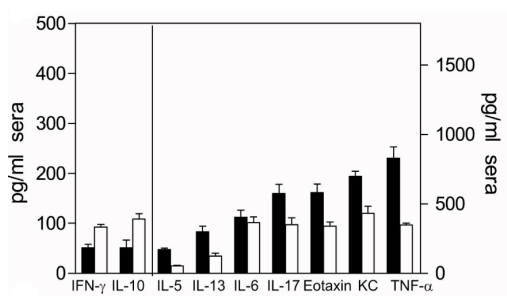

(a)

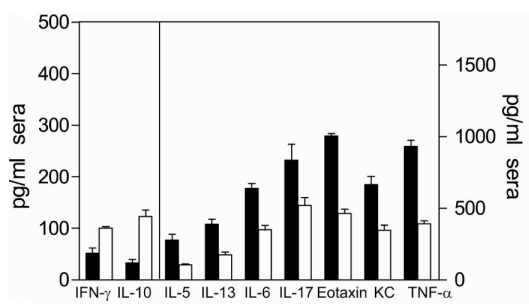

(c)

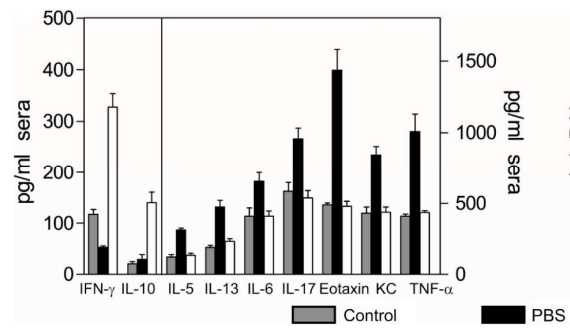

(e)

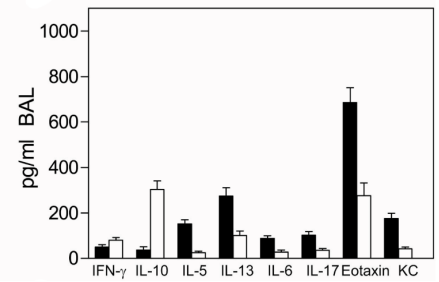

(b)

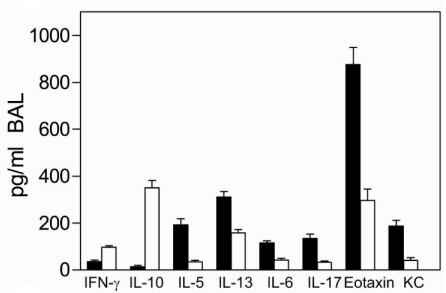

(d)

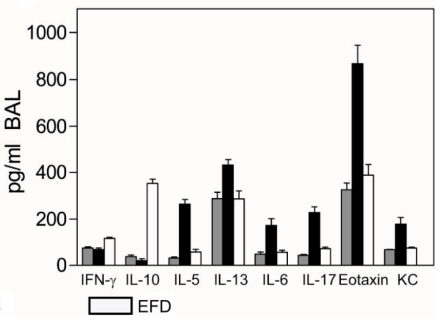

(f)

Figure 5. EFD BCG reduces inflammatory cytokines in BAL and sera in a chronic model of asthma. OVA-sensitized mice (6 per group) were PBS- or EFD BCG-treated after 1 (a), (b) 2 (c), (d) or 3 (e), (f) OVA-challenges and, cytokines were measured in BAL and sera collected 24 hours after the last OVA-challenges. Control mice were OVA-sensitized, PBS-treated after 3 OVA challenges and PBS-challenged (e), (f).

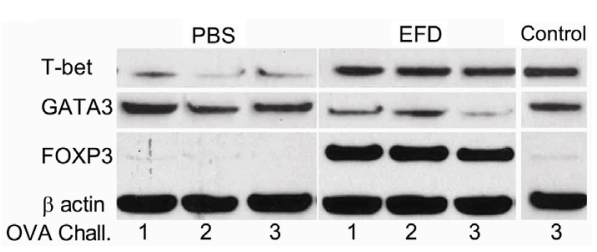

(a)
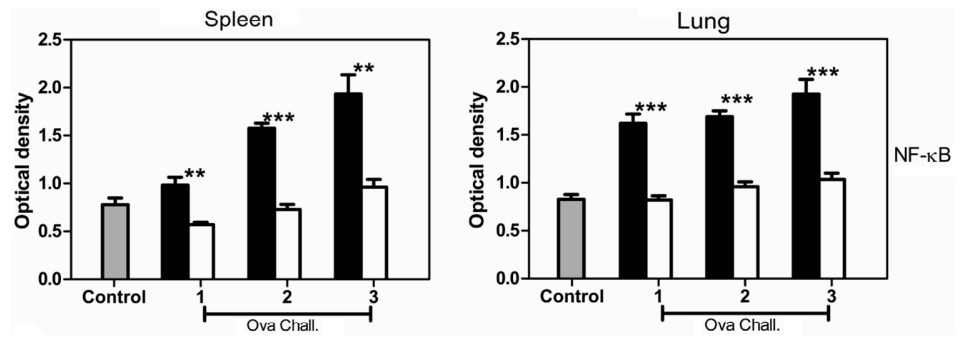

(b)
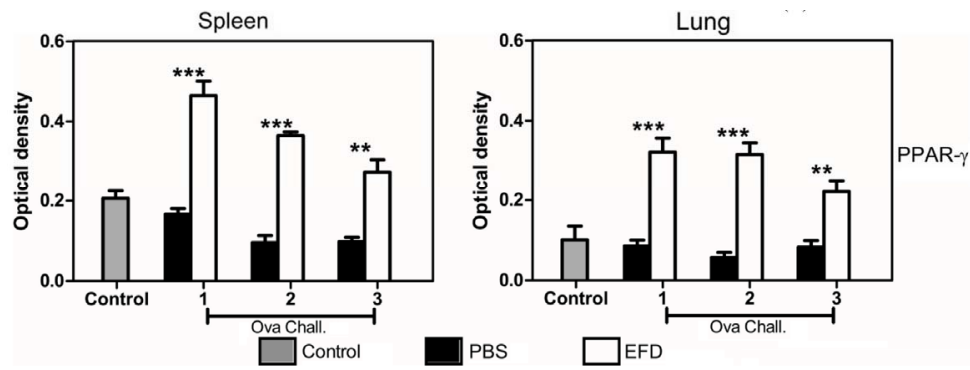

(c)

Figure 6. EFD BCG increases Foxp3, PPAR- $\gamma$ and reduces GATA-3 and NF- $\boldsymbol{k}$ B transcription factors in a chronic model of asthma. (a) T-bet, GATA-3 and Foxp3 transcription factor expression in lung cell extracts from PBS- or EFD BCG-treated mice after 1, 2, or 3 OVA challenges. Each band represents lung protein extracts of 2 mice; (b) NF- $\kappa$ B and (c) PPAR- $\gamma$ transcription factor expression in nuclear lung cell extracts from PBS- or EFD BCG-treated mice after 1, 2, or 3 OVA-challenges (6 mice per group). ${ }^{* *} \mathrm{P}<0.01,{ }^{* * *} \mathrm{P}<0.001$. 
time. EFD BCG could be such an alternative treatment since it reduces lung inflammation through long-term homing of Tregs, IL-10 and IFN- $\gamma$ production and NF- $\kappa \mathrm{B}$ blockade.

\section{Acknowledgements}

The authors would like to express their sincere thanks to Dr. Mohamad Abolhassani for his kind help with the Western-Blots and AHR. We thank Dr. Michel Huerre for lung histology.

\section{REFERENCES}

[1] W. W. Busse, S. Banks-Schlegel and S. E. Wenzel, "Pathophysiology of Severe Asthma," Journal of Allergy and Clinical Immunology, Vol. 106, No. 6, 2000, pp. 10331042. doi:10.1067/mai.2000.111307

[2] P. Pala, S. D. Message, S. L. Johnston and P. J. Openshaw, "Increased Aeroallergen-Specific Interleukin-4-Producing T Cells in Asthmatic Adults," Clinical and Experimental Allergy, Vol. 32, No. 12, 2002, pp. 1739-1744. doi:10.1046/j.1365-2222.2002.01548.x

[3] N. Krug, T. Jung, U. Napp, K. Wagner, G. SchultzeWerninghaus, C. Heusser, C. H. Rieger, U. Schauer and H. Fabel, "Frequencies of T Cells Expressing Interleukin4 and Interleukin-5 in Atopic Asthmatic Children. Comparison with Atopic Asthmatic Adults," American Journal of Respiratory and Critical Care Medicine, Vol. 158, No. 3, 1998, pp. 754-759. doi:10.1164/ajrccm.158.3.9507038

[4] T. Kiwamoto, Y. Ishii, Y. Morishima, K. Yoh, A. Maeda, K. Ishizaki, T. Iizuka, A. E. Hegab, Y. Matsuno, S. Homma, A. Nomura, T. Sakamoto, S. Takahashi and K. Sekizawa, "Transcription Factors T-Bet and GATA-3 Regulate Development of Airway Remodeling," American Journal of Respiratory and Critical Care Medicine, Vol. 174, No. 2, 2006, pp. 142-151. doi:10.1164/rccm.200601-0790C

[5] C. Osterlund, H. Gronlund, N. Polovic, S. Sundstrom, G. Gafvelin and A. Bucht, "The Non-Proteolytic House Dust Mite Allergen Der p 2 Induce NF-kappaB and MAPK Dependent Activation of Bronchial Epithelial Cells," Clinical and Experimental Allergy, Vol. 39, No. 8, 2009, pp. 1199-1208. doi:10.1111/j.1365-2222.2009.03284.X

[6] P. J. Barnes and I. M. Adcock, "Glucocorticoid Resistance in Inflammatory Diseases," Lancet, Vol. 373, No. 9678, 2009, pp. 1905-1917. doi:10.1016/S0140-6736(09)60326-3

[7] M. Spears, I. Donnelly, L. Jolly, M. Brannigan, K. Ito, C. McSharry, J. Lafferty, R. Chaudhuri, G. Braganza, P. Bareille, L. Sweeney, I. M. Adcock, P. J. Barnes, S. Wood and N. C. Thomson, "Bronchodilatory Effect of the PPAR-Gamma Agonist Rosiglitazone in Smokers with Asthma," Clinical Pharmacology \& Therapeutics, Vol. 86, No. 1, 2009, pp. 49-53. doi:10.1038/clpt.2009.41

[8] C. M. Hawrylowicz, "Regulatory T Cells and IL-10 in Allergic Inflammation," Journal of Experimental Medicine, Vol. 202, No. 11, 2005, pp. 1459-1463.

\section{doi:10.1084/jem.20052211}

[9] M. Lagranderie, M. Abolhassani, J. A. Vanoirbeek, C. Lima, A. M. Balazuc, B. B. Vargaftig and G. Marchal, "Mycobacterium bovis Bacillus Calmette-Guerin Killed by Extended Freeze-Drying Targets Plasmacytoid Dendritic Cells to Regulate Lung Inflammation," Journal of Immunology, Vol. 184, No. 2, 2010, pp. 1062-1070. doi:10.4049/jimmunol.0901822

[10] M. Gheorghiu, P. H. Lagrange, M. Lagranderie and A. M. Balazuc, "The Effects of Dispersed or Surface Grown Cultures, Manufacture and Control Methods on BCG Standardization," Development in Biological Standardization, Vol. 58, No. Pt A, 1986, pp. 191-205.

[11] M. Lagranderie, M. Abolhassani, J. Vanoirbeek, J. Lefort, M. A. Nahori, E. S. J. R. Lapa, M. Huerre, B. Vargaftig and G. Marchal, "Mycobacterium bovis BCG Killed by Extended Freeze-Drying Reduces Airway Hyperresponsiveness in 2 Animal Models," Journal of Allergy and Clinical Immunology, Vol. 121, No. 2, 2008, pp. 471- 478. doi:10.1016/j.jaci.2007.09.033

[12] J. A. Vanoirbeek, M. Rinaldi, V. De Vooght, S. Haenen, S. Bobic, G. Gayan-Ramirez, P. H. Hoet, E. Verbeken, M. Decramer, B. Nemery and W. Janssens, "Noninvasive and Invasive Pulmonary Function in Mouse Models of Obstructive and Restrictive Respiratory Diseases," American Journal of Respiratory Cell and Molecular Biology, Vol. 42, No. 1, 2010, pp. 96-104. doi:10.1165/rcmb.2008-04870C

[13] M. Tarkowski, J. A. Vanoirbeek, H. M. Vanhooren, V. De Vooght, C. M. Mercier, J. Ceuppens, B. Nemery and P. H. Hoet, "Immunological Determinants of Ventilatory Changes Induced in Mice by Dermal Sensitization and Respiratory Challenge with Toluene Diisocyanate," American Journal of Physiology-Lung Cellular and Molecular Physiologyl, Vol. 292, No. 1, 2007, pp. L207-214.

[14] D. Agnello, C. S. Lankford, J. Bream, A. Morinobu, M. Gadina, J. J. O'Shea and D. M. Frucht, "Cytokines and Transcription Factors that Regulate T Helper Cell Differentiation: New Players and New Insights," Journal of Clinical Immunology, Vol. 23, No. 3, 2003, pp. 147-161. doi:10.1023/A:1023381027062

[15] M. Lagranderie, C. Kluge, H. Kiefer-Biasizzo, M. Abolhassani, M. A. Nahori, C. Fitting, M. Huerre, A. Bandeira, H. Bercovier and G. Marchal, "Mycobacterium bovis Bacillus Calmette-Guerin Killed by Extended Freeze-Drying Reduces Colitis in Mice," Gastroenterology, Vol. 141, No. 2, 2011, pp. 642-652, 652, e641-644.

[16] D. J. Evans, P. Cullinan and D. M. Geddes, "Cyclosporin as an Oral Corticosteroid Sparing Agent in Stable Asthma," Cochrane Database of Systematic Reviews, No. 2, 2001, Article ID: CD002993.

[17] E. Xystrakis, S. Kusumakar, S. Boswell, E. Peek, Z. Urry, D. F. Richards, T. Adikibi, C. Pridgeon, M. Dallman, T. K. Loke, D. S. Robinson, F. J. Barrat, A. O'Garra, P. Lavender, T. H. Lee, C. Corrigan and C. M. Hawrylowicz, "Reversing the Defective Induction of IL-10-Secreting Regulatory T Cells in Glucocorticoid-Resistant Asthma Patients," Journal of Clinical Investigation, Vol. 116, No. 1, 2006, pp. 146-155. doi:10.1172/JCI21759 
[18] K. Asadullah, W. Sterry and H. D. Volk, "Interleukin-10 therapy-Review of a New Approach," Pharmacological Reviews, Vol. 55, No. 2, 2003, pp. 241-269. doi:10.1124/pr.55.2.4

[19] K. J. Erb, J. W. Holloway, A. Sobeck, H. Moll and G. Le Gros, "Infection of Mice with Mycobacterium bovis-Bacillus Calmette-Guerin (BCG) Suppresses Allergen-Induced Airway Eosinophilia," Journal of Experimental Medicine, Vol. 187, No. 4, 1998, pp. 561-569. doi:10.1084/jem.187.4.561

[20] M. A. Nahori, M. Lagranderie, J. Lefort, F. Thouron, D. Joseph, N. Winter, B. Gicquel, J. R. Lapa e Silva and B. B. Vargaftig, "Effects of Mycobacterium bovis BCG on the Development of Allergic Inflammation and Bronchial Hyperresponsiveness in Hyper-IgE BP2 Mice Vaccinated as Newborns," Vaccine, Vol. 19, No. 11-12, 2001, pp. 1484-1495. doi:10.1016/S0264-410X(00)00345-5

[21] T. Major, G. Wohlleben, B. Reibetanz and K. J. Erb, "Application of Heat Killed Mycobacterium bovis-BCG into the Lung Inhibits the Development of Allergen-Induced Th2 Responses," Vaccine, Vol. 20, No. 11-12, 2002, pp. 1532-1540. doi:10.1016/S0264-410X(01)00496-0

[22] P. Stock, O. Akbari, G. Berry, G. J. Freeman, R. H. Dekruyff and D. T. Umetsu, "Induction of T Helper Type 1-Like Regulatory Cells that Express Foxp3 and Protect against Airway Hyper-Reactivity," Nature Immunology,
Vol. 5, No. 11, 2004, pp. 1149-1156. doi:10.1038/ni1122

[23] S. J. Szabo, S. T. Kim, G. L. Costa, X. Zhang, C. G. Fathman and L. H. Glimcher, "A Novel Transcription Factor, T-Bet, Directs Th1 Lineage Commitment," Cell, Vol. 100, No. 6, 2000, pp. 655-669. doi:10.1016/S0092-8674(00)80702-3

[24] H. Hammad, H. J. de Heer, T. Soullie, V. Angeli, F. Trottein, H. C. Hoogsteden and B. N. Lambrecht, "Activation of Peroxisome Proliferator-Activated Receptor-Gamma in Dendritic Cells Inhibits the Development of Eosinophilic Airway Inflammation in a Mouse Model of Asthma," American Journal of Pathology, Vol. 164, No. 1, 2004, pp. 263-271.

doi:10.1016/S0002-9440(10)63116-1

[25] L. Klotz, I. Dani, F. Edenhofer, L. Nolden, B. Evert, B. Paul, W. Kolanus, T. Klockgether, P. Knolle and L. Diehl, "Peroxisome Proliferator-Activated Receptor Gamma Control of Dendritic Cell Function Contributes to Development of CD4 + T Cell Anergy," Journal of Immunology, Vol. 178, No. 4, 2007, pp. 2122-2131.

[26] D. Hartl, B. Koller, A. T. Mehlhorn, D. Reinhardt, T. Nicolai, D. J. Schendel, M. Griese and S. KraussEtschmann, "Quantitative and Functional Impairment of Pulmonary CD4 + CD25hi Regulatory T Cells in Pediatric Asthma," Journal of Allergy and Clinical Immunology, Vol. 119, No. 5, 2007, pp. 1258-1266. doi:10.1016/j.jaci.2007.02.023 


\section{Appendix}

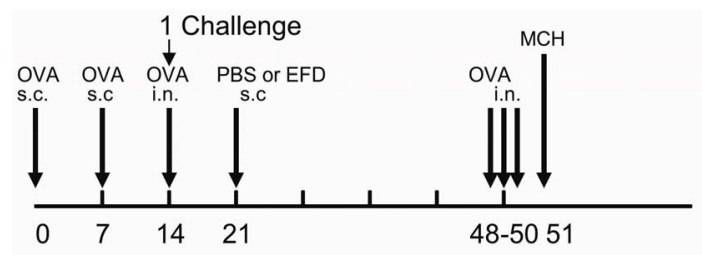

(a)

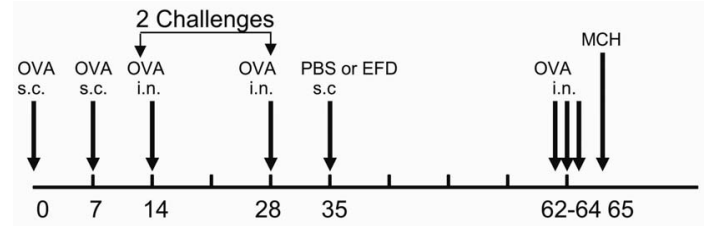

(b)

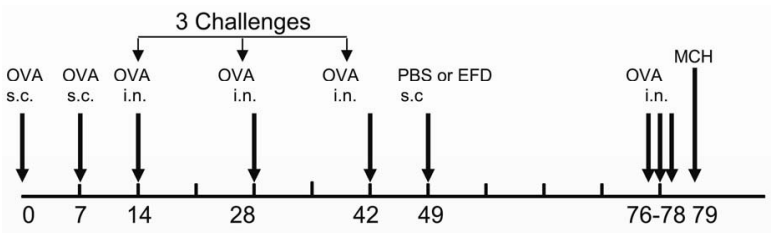

(c)

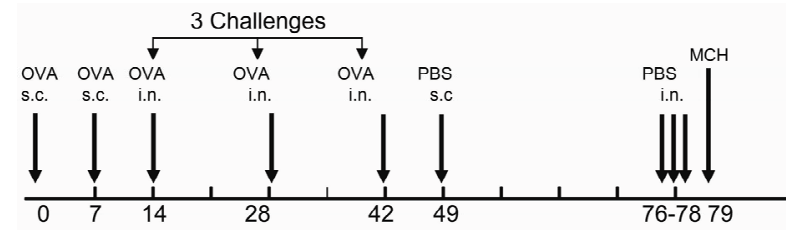

(d)

Supplementary Figure 1. Protocol of OVA sensitization and challenges in a chronic model of asthma (time in days). 